\title{
Effect of exercise on the maternal outcome in pregnancy of spontaneously hypertensive rats ${ }^{1}$
}

\author{
Renato Rocha ${ }^{\mathrm{I}}$, José Carlos Peraçoli ${ }^{\mathrm{II}}$, Gustavo Tadeu Volpato ${ }^{\mathrm{III}}$, Débora Cristina Damasceno ${ }^{\mathrm{IV}}$, Kleber Eduardo de Campos ${ }^{\mathrm{III}}$ \\ DOI: http://dx.doi.org/10.1590/S0102-8650201400150002 \\ IFellow PhD degree, Postgraduate Program in Obstetrics, Gynecology and Mastology, Botucatu Medical School, UNESP, Botucatu-SP, Brazil. \\ Acquisition and interpretation of data, manuscript preparation. \\ IIPhD, Full Professor, Department of Gynecology and Obstetrics, Botucatu Medical School, State University of Sao Paulo (UNESP), Botucatu-SP, \\ Brazil. Conception, design, intellectual and scientific content of the study.

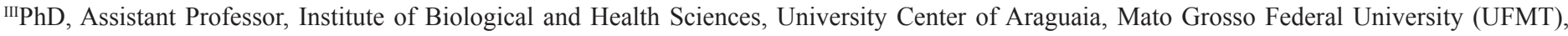 \\ Brazil. Critical revision. \\ ${ }^{\text {IV }} \mathrm{PhD}$, Researcher, Laboratory, Experimental Research on Gynecology and Obstetrics, Botucatu Medical School, UNESP, Botucatu-SP, Brazil. Critical \\ revision.
}

\begin{abstract}
PURPOSE: To evaluate the effect of exercise (swimming) on pregnancy in spontaneously hypertensive rats (SHR).

METHODS: Thirty three pregnant female SHR were distributed into three groups ( $\mathrm{n}=11$ animals/group): SHR Control=non-exercised (sedentary); SHR Ex $0=$ exercised from day zero to day 20 of pregnancy; and SHR Ex 7 = exercised from day 7 to 20 of pregnancy. Body weight and systolic blood pressure were indirectly measured during pregnancy. On gestational day 21 , the rats were anaesthetized and uterine content was withdrawn for analysis of maternal reproductive outcome parameters and fetal development.

RESULTS: The reduced blood pressure percentage was higher in SHR Ex0 and SHR Ex7 compared to SHR Control group. Weight gain was present in all pregnancy periods, but it was lower in SHR Ex7 than in SHR Control dams. The exercise increased the preimplantation loss rate. The post-implantation loss rate was lower in SHR Ex0 group. SHR Ex7 group showed a significantly higher percentage of fetuses classified as small for gestational age as compared to others groups.
\end{abstract}

CONCLUSION: The exercise contributed to lowering gestational blood pressure in SHR rats, but had a negative impact on the developing embryo.

Key words: Exercise. Hypertension. Pregnancy. Rats, Inbred SHR. 


\section{Introduction}

Hypertensive disorders represent the most common medical complications of pregnancy, with a reported incidence up to $10 \%$ of all gestations ${ }^{1}$. These disorders are a major cause of maternal and perinatal mortality and morbidity worldwide ${ }^{2}$. The term hypertension in pregnancy is commonly used to describe a wide spectrum of patients who may have only mild elevations in blood pressure or severe hypertension with dysfunctions of various organs. The hypertensive disorders may be caused by pregnancy (preeclampsia) or in result from different underlying factors such as chronic hypertension and renal disease ${ }^{3}$

It is estimated that $3 \%$ of pregnant women in the United States have chronic hypertension ${ }^{4}$. Chronic hypertension in pregnancy is associated with increased adverse maternal and fetal outcomes including superimposed preeclampsia, perinatal mortality, abrupt placentae, low birth weight, and intrauterine growth restriction (IUGR) $)^{5}$. It is estimated that about $10 \%$ to $25 \%$ of pregnant women with preexisting hypertension develop superimposed preeclampsia ${ }^{6}$.

The adoption of healthy lifestyles is critical for the prevention of high blood pressure (BP) and also it is an indispensable factor of the management of those with hypertension. Major lifestyle modifications shown to lower BP include physical activity. Also, regular and supervised physical activity decreases the BP of individuals with prehypertension and also stage 1 hypertension ${ }^{7}$. Literature shows that continuing regular, sustained exercise during pregnancy does not impair fetal well-being and provides multiple maternal benefits ${ }^{8}$. However, there were no reliable data about the condition of chronic hypertension during pregnancy. Guidelines suggest that women with complicated pregnancies should be discouraged from participating in exercise activities for fear of impacting the underlying disorder or maternal or fetal outcomes, for example, hypertensive pregnancy disorders ${ }^{9}$. The traditional medical advice has been for exercising women to reduce their habitual levels of effort in pregnancy and for non-exercising women to abstain from initiating strenuous exercise programs. This information was based on concerns that exercise could affect early and late pregnancy outcomes. However, there are promising reports of different experimental models used for research ${ }^{10-13}$.

The exercise-related maternal and fetal outcomes were already studied from normal pregnancies, usually involving athletes ${ }^{13,14}$, or studies comparing sedentary with physically active women during pregnancy ${ }^{15}$, or also comparing the physical effort in work environment and recreational activity ${ }^{16-18}$. There are no clear data about the association among pregnancy, chronic hypertension, and exercise; so that an experimental animal model becomes an important resource for evaluation of this issue. The most common experimental model of chronic hypertension is a strain of spontaneously hypertensive rats (SHR) ${ }^{19}$. Therefore, the present study aimed at evaluating an exercise (swimming) effect during pregnancy on spontaneously hypertensive rats and on their offspring.

\section{Methods}

The local Committee of Ethics in Animal Experimentation approved all experimental procedures of this study in accordance with the principles of the Guide for Care and Use of Experimental Animals.

Nine-week-old male and female SHR rats, weighing approximately $180 \mathrm{~g}$ and $220 \mathrm{~g}$ were kept in collective cages in controlled conditions of temperature of $\left(22 \pm 3^{\circ} \mathrm{C}\right)$, light $(12 \mathrm{~h}$ light/dark cycle) and relative humidity $(60 \pm 5 \%)$. The animals were fed with laboratory chow and tap water ad libitum. All female rats were mated overnight with SHR male rats. The day when sperm was found in the vaginal smear was designated gestational day 0 . The mating procedure consisted of 15 consecutive days, a period comprising approximately three estral cycles, until a replicate number of groups were obtained. However, during this period, unmated female rats were considered to be infertile and were discarded from the study ${ }^{20}$. Thirty three pregnant SHR were randomly distributed into three experimental groups $(n=11$ animals/group): SHR Control=non-exercised female SHR rats (sedentary); SHR Ex0=female SHR rats exercised from day zero to day 20 of pregnancy; and SHR Ex7=female SHR rats exercised from day 7 to 20 of pregnancy. The swimming program consisted of exercise model as described by Volpato et al. ${ }^{21}$ To familiarize the rats with the swimming system (water), they were daily exposed for $15 \mathrm{~min}$ for five days in a cage $(100 \mathrm{~cm}$ long, $70 \mathrm{~cm}$ wide, and $60 \mathrm{~cm}$ high) containing water at a depth of $10 \mathrm{~cm}$ at $32^{\circ} \mathrm{C}$. This period corresponded to the mating period. Pregnant rats that remained in this condition were classified as sedentary. Subsequently, the female rats familiarized with the swimming system were placed in a cage containing water at a depth of $40 \mathrm{~cm}$ deep. Exercise on the first day under these conditions lasted about $20 \mathrm{~min}$, with progressive increases of $10 \mathrm{~min}$ each day until the duration reached $60 \mathrm{~min}$. The rats were trained to swim for $1 \mathrm{~h}$ daily until the end of pregnancy period. Swimming was always performed at a water temperature of $32^{\circ} \mathrm{C}$ between 9 a.m. and 10 a.m. for six days a week.

The systolic blood pressure was measured indirectly without anesthesia, always in the morning, on days $0,7,14$ and 20 of the pregnancy period. The systolic pressures in 
the tail of the female rats were measured by use of a caudal plethysmograph with a polygraph (Byo-Sistem PE 300, NARCO), a sensor placed in the proximal region of the tail, along with an electrosphygmomanometer, to enable the recording of tail pressure. The animals were warmed at $37^{\circ} \mathrm{C}$ for 4 minutes and then transferred to an iron retainer, where the tail was exposed. In the proximal region of the tail, a sensor (KSM-microfone) was placed and coupled to the plethysmograph. Blood pressure was recorded on paper with the polygraph at a $2.5-\mathrm{mm} / \mathrm{s}$ velocity ${ }^{22}$. A value superior to 150 $\mathrm{mmHg}$ was considered the inclusion criterion for hypertension by mean of three consecutive measurements ${ }^{23}$. Body weight and food intake were weekly measured during whole pregnancy, at about in 9 a.m.

At day 21 of pregnancy, fed rats were anesthetized with sodium thiopental. Following trichotomy of the abdominal region, the animal was placed in dorsal decubitus, and its libs were fixed to the surgery table. The laparotomy procedure was carried out by an incision in the medium line beginning in the xiphoid cartilage and ending in the pubis. The intestinal loops were moved cranially for uterus exposure. The hysterectomy was carried out by ligament, artery and ovarian vein section and incision of the body uterine above the cervix. Afterward, the uterus and his content were weighed using analytical scale. Incisions were performed in the whole extension of the uterine horns, on their free margin and on the most avascular area. The fetus, amniotic sac and placenta were removed by slight traction. The umbilical cord was clamped by using Halsteadtype hemostatic clamps. The rate of embryonic loss before and after implantation was calculated ${ }^{24}$. If there was a lack of visible implantation sites, the uterine corns were stained with a preparation of $10 \%$ ammonium sulfate ${ }^{25}$. The fetuses were weighed and the mean birth weight of the SHR Control pups was $3.8 \pm 0.5 \mathrm{~g}$. Newborns in the experimental groups whose birth weights did not diverge more than \pm 1.0 standard deviation (SD) from the G1 mean (i.e., those that presented body weight from 3.3 to $4.3 \mathrm{~g}$ ) were classified as appropriate for pregnancy age (APA). Those whose weights were at least 1.0 SD greater than the G1 mean birth weight were classified as large for pregnancy age (LPA). Those whose birth weights were at least 1.0 SD lower than the G1 mean birth weight were classified as small for pregnancy age (SPA) ${ }^{26}$.

Analysis of variance (ANOVA) followed by Tukey's multiple comparison test was used to analyze mean values. The percentage values were calculated by Fisher's exact test. Differences were considered statistically significant when $p<0.05$.

\section{Results}

Maternal blood pressure during pregnancy

Figure 1 presents blood pressure (BP) data. It was verified that SHR Control group showed a reduction of blood pressure levels (BP) only at day 20 of pregnancy; groups SHR Ex0 and SHR Ex7 presented diminished BP at days 14 and 20 of pregnancy, showing a higher percentage of BP reduction in the exercised groups.

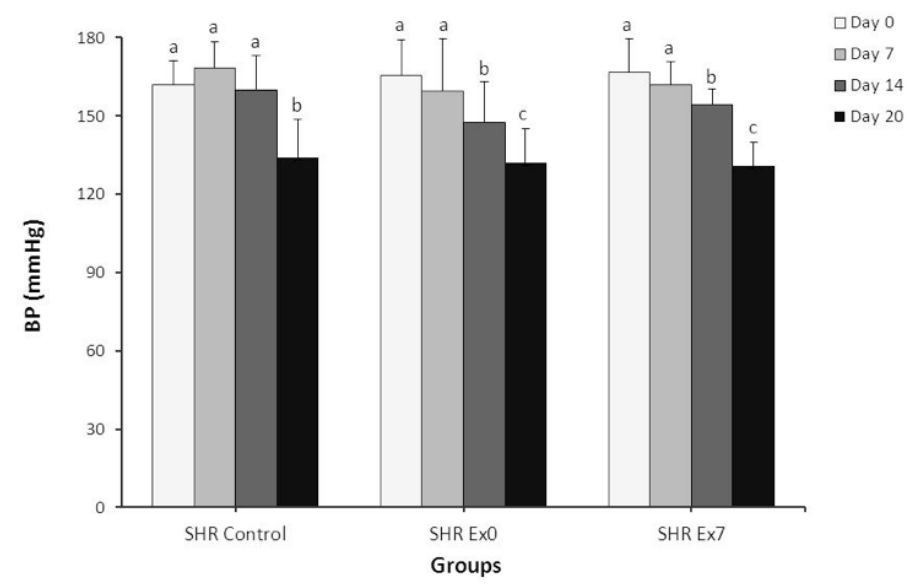

FIGURE 1 - Mean \pm standard deviation of systolic blood pressure $(\mathrm{mmHg})$ of spontaneously hypertensive rats (SHR) according to experimental group. Maternal body weight and food intake

Body weight values are shown in Figure 2. The rats from groups SHR Control and SHR Ex0 gained weight as a function of advancing pregnancy; and SHR Ex7 group presented increased body weight at days 14 and 21. Moreover, the total food intake (FI) did not differ among experimental groups. The ratio between food intake and weight gain (WG), percentage of weight gain and growth velocity also showed no statistically significant differences among experimental groups (Table 1).

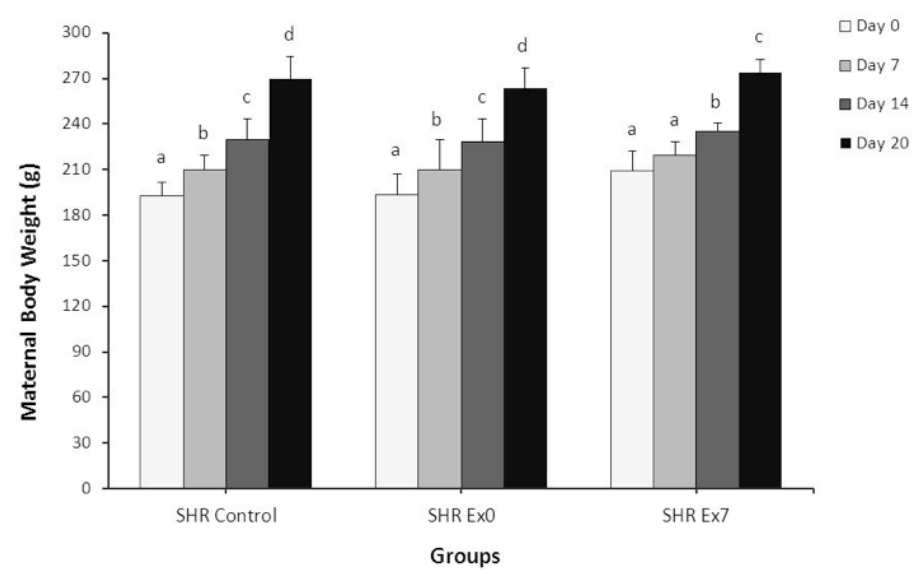

FIGURE 2 - Mean \pm standard deviation of maternal body weight (g) of spontaneously hypertensive rats (SHR) according to experimental group. 
TABLE 1 - Mean \pm standard deviation of total food intake (FI) (g), FI and weight gain (WG) ratio, percentage of WG and growth velocity $(\mathrm{GV})$ and FI/GV ratio in spontaneously hypertensive rats (SHR) from different experimental groups.

\begin{tabular}{cccc}
\hline \multicolumn{4}{c}{ Experimental groups } \\
& SHR Control & SHR Ex0 & SHR Ex7 \\
\hline Total FI & $429.8 \pm 42.0$ & $423.0 \pm 34.1$ & $422.9 \pm 35.8$ \\
FI/WG & $5.9 \pm 2.1$ & $6.3 \pm 1.8$ & $7.5 \pm 2.8$ \\
\%WG/GV & $15.9 \pm 5.1$ & $16.6 \pm 4.1$ & $19.9 \pm 6.6$ \\
FI/GV & $124.4 \pm 43.7$ & $132.8 \pm 36.7$ & $156.0 \pm 57.0$ \\
\hline
\end{tabular}

$\mathrm{p}>0.05$ - non significant difference

\section{Maternal reproductive outcomes}

The reproductive outcome data are shown in Table 2. The experimental groups did not differ significantly in the number of corpora lutea, implantations, live and dead fetuses, fetal and placental weight. The pre-implantation loss percentages of SHR Ex0 (28.02\%) and SHR Ex7 (32.16\%) were significantly higher compared to SHR Control (16.82\%); whereas the post-implantation loss rate was lower in SHR Ex0 (3.33\%) as compared with SHR Control (15.45\%) and SHR Ex7 (12.81\%). The percentage of fetuses classified as small for gestational age (SPA) was significantly higher in SHR Ex7 in relation to other experimental groups.

TABLE 2 - Maternal reproductive outcomes and fetal development parameters of spontaneously hypertensive rats (SHR) according to experimental group.

\begin{tabular}{lccc}
\hline \multicolumn{4}{c}{ Experimental groups } \\
& SHR Control & SHR Ex0 & SHR Ex7 \\
\hline Corpora Lutea & $13.3 \pm 1.3$ & $11.9 \pm 1.5$ & $13.4 \pm 2.6$ \\
Implantation & $11.0 \pm 2.0$ & $8.5 \pm 3.5$ & $8.8 \pm 3.4$ \\
Live Fetuses & $9.3 \pm 2.2$ & $8.1 \pm 3.1$ & $7.9 \pm 3.9$ \\
Dead Fetuses & $0.2 \pm 0.4$ & $0.0 \pm 0.0$ & $0.2 \pm 0.4$ \\
\% Preimplantation & 16.8 & $28.0 *$ & $32.2 *$ \\
loss & & & \\
\% Postimplantation & 15.4 & $3.3 *$ & 12.8 \\
loss & & & \\
Placental weight & $0.4 \pm 0.0$ & $0.4 \pm 0.1$ & $0.4 \pm 0.1$ \\
Fetal weight & $3.8 \pm 0.5$ & $3.7 \pm 0.5$ & $3.4 \pm 0.3$ \\
$\quad$ \% SPA & 12.6 & 15.0 & $23.9 *$ \\
\% APA & 76.8 & 73.8 & 75.0 \\
\% LPA & 10.6 & 11.2 & $1.1 *$ \\
\hline
\end{tabular}

Values represented as mean \pm standard deviation or percentage.

Legend: SPA = small for pregnancy age; APA = adequate for pregnancy age; LPA $=$ large for pregnancy age.

$* \mathrm{p}<0.05$ - significant statistically difference as compared to SHR Control group

\section{Discussion}

The interaction between the stress exposure caused by physical exercise in pregnant woman can lead to conflicts among the physiological needs from pregnancy, exerting its negative effects.

However, the literature presents the issue of exercise and pregnancy that has shown inexistent or favorable effects on both the mother and the fetus physiological factors. The benefits of regular physical activity during pregnancy include weight maintenance, reduced blood pressure, lower cardiovascular risk and diabetes control ${ }^{27}$.

There are few studies performed in humans that associate physical activity, hypertension and pregnancy, several of them are retrospective and use questionnaires to quantify the level of energy expenditure during free time. Studies report that physical activity decreases the risk of developing preeclampsia when performed at low-moderate intensity and less than 270 minutes per week ${ }^{28-32}$.

Our data showed reduction of blood pressure (BP) values in all groups during pregnancy. However, the decrease of BP was earlier and more pronounced in rats subjected to exercise. Experimental studies have produced controversial results on the behavior of $\mathrm{BP}$ during pregnancy in SHR models, probably due to slightly differences in the methodologies used to determine this parameter. Peraçoli et $a l .{ }^{33}$ observed that the BP values evaluated on alternating days was more accurate than weekly determinations, which showed that the reduction of BP at the end of pregnancy and the frequency of this also decreases stress by causing adaptation to the methodology. Moreover, Kvetnansky et al. ${ }^{34}$ using SHR models confirmed this fact, which could be explained by the lower release of catecholamines induced by immobilization stress during the experimental procedure. In the present study, the cathecolamine level was not determined, but the hypothesis of adaptation to stress can provide an explanation for the reduction of BP observed in all groups. Another explanation was animals were daily handled to be placed in a cage containing water regardless of the number of repeat blood pressure measurements. The fact that the animals in exercised groups decreased BP earlier and more markedly as compared with the sedentary rats can be attributed to the presence of physical exercise.

The experimental procedure of treating hypertensive pregnant woman by restricting physical activity and resting in the left lateral position is routine in prenatal clinics. This procedure is justified by the findings of decreases in preterm birth, BP values and the promotion of pressure diuresis ${ }^{35}$. However, studies show that practice of aerobic physical exercise in water is beneficial for reducing swelling and increasing diuresis, thereby facilitating BP control $^{36,37}$. Regular physical exercise by hypertensive individuals 
is associated with beneficial effects on BP, neurohumoral release and autonomic activity. However, among the antihypertensive mechanisms of exercise that remain uncertain are the increased vascular bed $^{38}$, increased sensitivity of the baroreceptors ${ }^{39}$, increased formation and action of antioxidants ${ }^{40}$ and also the modulation of sympathetic and parasympathetic tone ${ }^{41}$.

The effects of exercise on BP are dependent on the type, duration and intensity of the protocol used ${ }^{42}$. Véras-Silva et al. ${ }^{43}$ showed that after physical exercise of low intensity, the values of BP in SHR were lower in rats of the similar strain as compared with those that were sedentary or practiced high-intensity exercise. According to Forjaz et $a l .{ }^{44}$ this hypotensive response is more associated with decreased cardiac sympathetic activity rather than changes in vagal tone or intrinsic heart rate.

The total body weight of rats during pregnancy was similar among the exercised groups, increasing weekly until the end of pregnancy. However, SHR Ex7 rats presented no increase in the body weight at the first week of the pregnancy, related to non-exercising period of this group. The analysis of food intake and its relationship with weight gain, percentage weight gain and growth rate showed no significant differences among the groups. Physical exercise practiced since the beginning of pregnancy may have contributed to lack of weight gain found in SHR Ex7 rats.

In the present study we observed that the rate of embryonic loss before implantation was higher in rats that performed exercise whereas the post-implantation loss was lower in the group that practiced the exercise from the day 7 of pregnancy. Therefore, exercise is a predisposing factor for early fetal loss (pre- and post-implantation), once the rats were adapted the exercise programming in the first seven days of pregnancy the incidence of fetal loss was significantly lower.

The effects of hypertension on the fetus are well known. The maternal BP levels are inversely related to the size and weight of the newborn ${ }^{19}$. These effects are caused by uteroplacental hypoperfusion consequent to high $\mathrm{BP}^{45}$. The analysis of perinatal outcomes indicates that exercise did not affect the litter mean weight as compared with sedentary rats. However, the exercising program in pregnancy had a higher percentage of fetuses defined as small for gestation age. This fact might be justified by the interference in the adaptation of the maternal organism, which impaired the fetal development.

\section{Conclusion}

The exercise contributed to lowering gestational blood pressure in SHR rats, but had a negative impact on the developing embryo.

\section{References}

1. ACOG Committee on Practice Bulletins-Obstetrics. ACOG practice bulletin: Diagnosis and management of preeclampsia and eclampsia. Int J Gynaecol Obstet. 2002;99:159-67. doi: 10.1016/ S0020-7292(02)80002-9.

2. Sibai BM. Diagnosis and management of gestational hypertension and preeclampsia. Obstet Gynecol. 2003;102:181. doi: 10.1016/ S0029-7844(03)00475-7.

3. ACOG Committee on Obstetric Practice. ACOG practice bulletin. Diagnosis and management of preeclampsia and eclampsia. Int J Gynaecol Obstet. 2002;77(1):67-75. doi: 10.1016/S00207292(02)80002-9.

4. Walther T, Wessel N, Baumert M, Stepan H, Voss A, Faber R. Longitudinal analysis of heart rate variability in chronic hypertensive pregnancy. Hypertens Res. 2005;28:113-8. doi: 10.1291/hypres.28.113.

5. Sibai BM. Chronic hypertension in pregnancy. Obstet Gynecol. 2002;100:369-77. doi: 10.1161/circulationaha.106.636472.

6. Gilbert WM, Young AL, Danielsen B. Pregnancy outcomes in women with chronic hypertension: a population-based study. J Reprod Med. 2007;52:1046-51. PMID:18161404.

7. Chobanian AV, Bakris GL, Black HR, Cushman WC, Green LA, Izzo JL Jr, Jones DW, Materson BJ, Oparil S, Wright JT Jr, Roccella EJ, Joint National Committee on Prevention, Detection, Evaluation, and Treatment of High Blood Pressure. National Heart, Lung, and Blood Institute; National High Blood Pressure Education Program Coordinating Committee. Seventh report of the joint national committee on prevention, detection, evaluation, and treatment of high blood pressure. Hypertension. 2003;42:1206-52. doi: 10.1001/ jama.289.19.2560.

8. Clapp III JF. Exercise during pregnancy: a clinical update. Clin Sport Med. 2000;19:273-86. doi: 10.1016/S0278-5919(05)70203-9.

9. Davies GAL, Wolfe LA, Mottola MF, MacKinnon C. Joint SOGC/CSEP Clinical Practice Guideline: Exercise in pregnancy and the postpartum period. Can J Appl Physiol. 2003;28:329-41. PMID: 12955862.

10. Clapp III JF, Kim H, Burciu B, Schmidt S, Petry K, Lopez B. Continuing regular exercise during pegnancy: Effect of exercise volume on fetoplacental growth. Am J Obstet Gynecol. 2002;186:142-7. doi: 10.1067/mob.2002.119109.

11. Clapp III JF, Lopez B, Harcar-Sevcik R. Neonatal behavioral profile of the offspring of women who continued to exercise regularly throughout pregnancy. Am J Obstet Gynecol. 1999;180:91-4. doi: 10.1016/S0002-9378(99)70155-9.

12. Kardel KR, Kase T. Training during pregnancy: effects on fetal development and birth. Am J Obstet Gynecol. 1998;178:280-6. doi: 10.1016/S0002-9378(98)80013-6.

13. Bailey DM, Davies B, Budgett R, Sanderson DC, Griffin D. Endurance training during a twin pregnancy in a marathon runner. Lancet. 1998;351:1182-3. doi: 10.1016/S0140-6736(05)79128-5.

14. Clapp III JF. Morphometric and neurodevelopmental outcome at age five years of the offspring of women who continued to exercise regularly throughout pregnancy. J Pediatr. 1996;129:856-63. doi: 10.1016/S0022-3476(96)70029-X

15. Clapp III JF, Simonian S, Lopez B, Appleby-Wineberg S, HarcarSevcik R. The one-year morphometric and neurodevelopmental outcome of the offspring of women who continued to exercise regularly throughout pregnancy. Am J Obstet Gynecol. 1998;178:594-9. doi:10.1016/S0002-9378(98)70444-2.

16. Higgins JR, Walshe JJ, Conroy RM, Darling MRN. The relation between maternal work, ambulatory blood pressure, and pregnancy hypertension. J Epidemiol Community Health. 2002;56:389-93. doi:10.1136/jech.56.5.389. 
17. Campbell MK, Mottola MF. Recreational exercise and occupational activity during pregnancy and birth weight: a case-control study. Am J Obstet Gynecol. 2001;184:403-8. doi: 10.1067/mob.2001.109392.

18. Luke BSCD, Avni MBA, Min LMPH, Misiunas RBA. Work and pregnancy: The rule of fatigue and the "second shift" on antenatal morbidity. Am J Obstet Gynecol. 1999;181:1172-9. doi: 10.1016/ S0002-9378(99)70103-1.

19. Ahokas RA, Sibai BM. The relationship between experimentally determined litter size and maternal blood pressure in spontaneously hypertensive rats. Am J Obstet Gynecol. 1990;162:841-7. doi: 10.1016/0002-9378(90)91021-4.

20. Damasceno DC, Silva HP, Vaz GF, Vasques-Silva FA, Calderon IM, Rudge MV, Campos KE, Volpato GT. Diabetic rats exercised prior to and during pregnancy: maternal reproductive outcome, biochemical profile, and frequency of fetal anomalies. Reprod Sci. 2013;20:7308. doi: $10.1177 / 1933719112461186$.

21. Volpato GT, Damasceno DC, Kempinas WG, Rudge MVC, Calderon IMP. Effect of exercise on the reproductive outcome and fetal development of diabetic rats. Reprod Biomed Online. 2009;19:8528. doi: 10.1016/j.rbmo.2009.09.027.

22. Castardeli E, Paiva, SRA, Matsubara BB, Matsubara LS, Minicucci MF, Azevedo PS, Campana AO, Zornoff LA. Chronic cigarette smoke exposure results in cardiac remodeling and impaired ventricular function in rats. Arq Bras Cardiol. 2005;84:320-4. doi: 10.1590/S0066-782X2005000400009.

23. Okamoto K, Aoki K. Development of a strain of spontaneously hypertensive rats. Jpn Circ J. 1963;7:282-93. PMID: 13939773.

24. Damasceno DC, Kiss ACI, Sinzato YK, Campos KE, Rudge MVC, Calderon IMP, Volpato GT. Maternal-fetal outcome, lipid profile and oxidative stress of diabetic rats neonatally exposed to streptozotocin. Exp Clin Endocrinol Diabetes. 2011;119:408-13. doi: $10.1055 / \mathrm{s}-0030-1269886$.

25. Salewsky E. Farbemethode zum markroskopishen nachweis von implantatconsstellen na uterus der ratter naunyn schmuderbergs. Arch Pharm. 1964;247:367.

26. Volpato GT, Calderon IM, Sinzato S, Campos KE, Rudge MV, Damasceno DC. Effect of Morus nigra aqueous extract treatment on the maternal-fetal outcome, oxidative stress status and lipid profile of streptozotocin-induced diabetic rats. J Ethnopharmacol. 2011;138:691-6. doi: 10.1016/j.jep.2011.09.044.

27. Weissgerber TL, Wolfe LA, Davies GAL, Mottola MF. Exercise in the prevention and treatment of maternal-fetal disease: a review of the literature. Appl Physiol Nutr Metab. 2006;31:661-74. PMID: 17213880 .

28. Yeo S. Adherence to walking or stretching, and risk of preeclampsia in sedentary pregnant women. Res Nurs Health. 2009;32:379-90. doi: 10.1002/nur.20328.

29. Østerdal M, Strøm M, Klemmensen A, Knudsen V, Juhl M, Halldorsson TI, Nybo Andersen AM, Magnus P, Olsen SF. Does leisure time physical activity in early pregnancy protect against pre-eclampsia? Prospective cohort in Danish women. BJOG. 2009;116:98-107. doi: 10.1111/j.1471-0528.2008.02001.x.

30. Bovbjerg ML, Siega-Riz AM. Exercise during pregnancy and cesarean delivery: North Carolina PRAMS, 2004-2005. Birth. 2009;36:200-7. doi: 10.1111/j.1523-536X.2009.00324.x.

31. Rudra CB, Sorensen TK, Luthy DA, Williams MA. A Prospective analysis of recreational physical activity and preeclampsia risk. Med Sci Sports Exerc. 2008;40:1581-8. doi: 10.1249/ MSS.0b013e31817cab1.

32. Saftlas AF, Logsden-Sackett N, Wang W, Woolson R, Bracken MB. Work, leisure-time physical activity, and risk of preeclampsia and gestational hypertension. Am J Epidemiol. 2004;160:758-65. doi: 10.1093/aje/kwh277.
33. Peraçoli JC, Rudge MVC, Sartori MS, Franco RJS. Effects of hypertension on maternal adaptations to pregnancy: experimental study on spontaneously hypertensive rats. Sao Paulo Med J. 2001;119:54-8. doi.org/10.1590/S1516-31802001000200003.

34. Kvetnansky R, McCarty R, Thoa NB, Lake CR, Kopin IJ. Sympathoadrenal responses of spontaneously hypertensive rats to immobilization stress. Am J Physiol. 1979;25:H457-62.

35. Lenfant C. Working group report on high blood pressure in pregnancy. J Clin Hypertens. 2001;3:75-8.

36. Coelho BT, Polito MD. Acute effect of a water aerobics session on the response of blood pressure in hypertensive non-pregnant women. Rev SOCERJ. 2009;22:75-9.

37. Kent T, Gregor J, Deardorff L, Katz V. Edema of pregnancy: a comparison of water aerobics and static immersion. Obstet Gynecol. 1999;94:726-9. PMID: 10546718.

38. Amaral SL, Silveira NP, Zorn TMT, Michelini LC. Exercise training causes skeletal muscle venular growth and alters hemodynamic responses in spontaneously hypertensive rats. J Hypertens. 2001;19:931-40. PMID: 11393677.

39. Brum PC, Da Silva GJJ, Moreira ED, Ida F, Negrão CE, Krieger EM. Exercise training increases baroreceptor gain sensitivity in normal and hypertensive rats. Hypertension. 2000;36:1018-22. doi: 10.1161/01.HYP.36.6.1018.

40. Jonsdottir IH, Jungersten L, Johansson C, Wennmalm A, Thorén $\mathrm{P}$, Hoffmann P. Increase in nitric oxide formation after chronic voluntary exercise in spontaneously hypertensive rats. Acta Physiol Scand. 1998;162:149-53. doi: 10.1046/j.1365-201X.1998.0285f.x.

41. Krieger EM, Brum PC, Negrão CE. Role of arterial baroreceptor function on cardiovascular adjustments to acute and chronic dynamic exercise. Biol Res. 1998;31:273-9. PMID: 9830515.

42. Viecili PR, Bündchen DC, Richter CM, Dipp T, Lamberti DB, Pereira AM, Barbosa L de C, Rubin AC, Barbosa EG, Panigas TF. Doseresponse curve to exercise in hypertensive individuals: analysis of the number of sessions to the hypotensive effect. Arq Bras Cardiol. 2009;92:361-7. doi: 10.1590/S0066-782X2009000500010.

43. Verás-Silva AS, Matto KC, Gava NS, Brum PC, Negrão CE, Krieger EM. Low-intensity exercise training decreases cardiac output and hypertension in spontaneously hypertensive rats. Am J Physiol. 1997;273:H2627-31. PMID: 9435596.

44. Forjaz CLM, Rondon MUPB, Negrão CE. Efeitos hipotensores e simpatolíticos do exercício aeróbio na hipertensão arterial. Rev Bras Hipertens. 2005;12:245-50. PMID 427047.

45. Chiang CE, Wang TD, Li YH, Lin TH, Chien KL, Yeh HI, Shyu KG, Tsai WC, Chao TH, Hwang JJ, Chiang FT, Chen JH; Hypertension Committee of the Taiwan Society of Cardiology. 2010 Guidelines of the Taiwan Society of Cardiology for the management of hypertension. J Formos Med Assoc. 2010;109:740-73. doi: 10.1016/ S0929-6646(10)60120-9.

\section{Correspondence:}

Prof. Dr. José Carlos Peraçoli

Departamento de Ginecologia e Obstetrícia

Faculdade de Medicina de Botucatu-UNESP

Distrito de Rubião Júnior, $\mathrm{s} / \mathrm{n}$

18618-970 Botucatu - SP Brasil

Tel./Fax: (55 14)3811-6181

jperacoli@uol.com.br

jperacolifmb@gmail.com

Received: Apr 17, 2014

Review: Jun 18, 2014 
Accepted: July 21, 2014

Conflict of interest: none

Financial source: none

${ }^{1}$ Research performed at Laboratory of Experimental Research in Gynecology and Obstetrics, Department of Gynecology and Obstetrics, Botucatu Medical School, State University of Sao Paulo (UNESP), Botucatu-SP, Brazil. Part of PhD degree thesis, Postgraduate Program in Gynecology, Obstetrics and Mastology. Tutor: José Carlos Peraçoli. 